\title{
Benign Lymphoid Hyperplasia
}

National Cancer Institute

\section{Source}

National Cancer Institute. Benign Lymphoid Hyperplasia. NCI Thesaurus. Code C97078.

A polyclonal proliferation of lymphocytes without evidence of cytologic atypia. 